\title{
Congenital Cystic Adenomatoid Malformation of Lung: A Case Report
} Dr. Seena Susan Itty ${ }^{1 *}$, Dr. Sankar $S^{2}$

${ }^{1}$ Junior Resident, Department of Pathology, Government Medical College, Kottayam, Kerala, India
${ }^{2}$ Professor and HOD, Department of Pathology, Government Medical College, Kottayam, Kerala, India

DOI: $\underline{10.36348 / \mathrm{sjpm} .2022 . v 07 i 01.005}$

| Received: 06.12.2021 | Accepted: 18.01.2022 | Published: 26.01.2022

*Corresponding author: Dr. Seena Susan Itty

Junior Resident, Department of Pathology, Government Medical College, Kottayam, Kerala, India

\section{Abstract}

Congenital cystic adenomatoid malformation (CCAM), also known as Congenital Pulmonary Airway Malformation (CPAM) is a rare developmental anomaly of lung with unknown etiology. It is seen most commonly in the lungs of infants and has features of both immaturity and malformation of the small airways and distal lung parenchyma. With the increasing use of prenatal ultrasonography, cystic lung lesions are detected more often antenatally, which allows for better neonatal management. We report a case of 3 day old female baby presenting with respiratory distress soon after birth.

Keywords: Congenital Cystic Adenomatoid malformation (CCAM), Congenital Pulmonary Airway Malformation (CPAM), Lungs.

Copyright (C) 2022 The Author(s): This is an open-access article distributed under the terms of the Creative Commons Attribution 4.0 International License (CC BY-NC 4.0) which permits unrestricted use, distribution, and reproduction in any medium for non-commercial use provided the original author and source are credited.

\section{INTRODUCTION}

Congenital Cystic Adenomatoid Malformation (CCAM) is a rare abnormality of lung development with an incidence of 1 in 25,000 to 35,000 pregnancies which accounts for $25 \%$ of all congenital lung abnormalities and $95 \%$ of cases of congenital cystic lung disease. It is a hamartomatous lesion characterized by a cessation of normal bronchiolar maturation resulting in multicystic lesions in lung due to proliferation of the respiratory bronchioles. Most CCAMs occur in two distinct clinical settings: in a stillborn or extremely ill premature infant with anasarca or in a new born infant who rapidly develops respiratory distress that is related to an expanding mass in one hemithorax. Less often the lesion is seen in older children and a few cases have been reported in adults. The diagnosis of CCAM is made antenatally by obstetric ultrasound and postnatally by imaging radiography and MRI. In 1977, Stocker et al., classified CCAM into three histopathological groups. An expanded classification of five different types was further proposed in 2002. The pathogenesis of CCAM is unknown but is thought to be related to defective lung development due to embryological insult occurring before the $16^{\text {th }}$ week of intrauterine life. The mortality rate of prenatally diagnosed cases ranges from 9 to $49 \%$. The mainstay of treatment is surgical excision that prevents complications such as recurrent infections, pneumothorax and malignancy.

\section{CASE REPORT}

A 3 day old female baby, term/AGA/LSCS/CSAB, born out of wedlock to a primigravida woman was referred in view of worsening respiratory distress which developed soon after birth. Chest X-ray showed small left pneumothorax. CT Thorax showed small left pneumothorax, with a large $3.9 \times 2.7 \times 4.6 \mathrm{~cm}$ well defined thin walled air filled cyst in the left lower lobe of lung causing tracheamediastinal shift to right side and compressing right lung. Features were in favour of Type 1 Congenital Airway Malformation. On examination, baby was tachypnoeic with a respiratory rate of $80 /$ minute. Oxygen saturation was $85 \%$ in room air with hyperinflated chest, moderate subcostal retractions, decreased air entry on left side which was hyperresonant on percussion and apex beat shifted toward sternal margin. Left posterolateral thoracotomy and left lower lobectomy was done in view of worsening respiratory distress.

Single lobe of lung specimen in formalin was received weighing 20 gram and measuring $6 \times 6 \times 3 \mathrm{~cm}$. Posterior surface showed a bleb measuring $2 \mathrm{~cm}$ in 
greatest dimension. Cutting through hilum identified a unilocular cyst measuring $4 \mathrm{~cm}$ in greatest dimension which extruded dark brown hemorrhagic fluid.

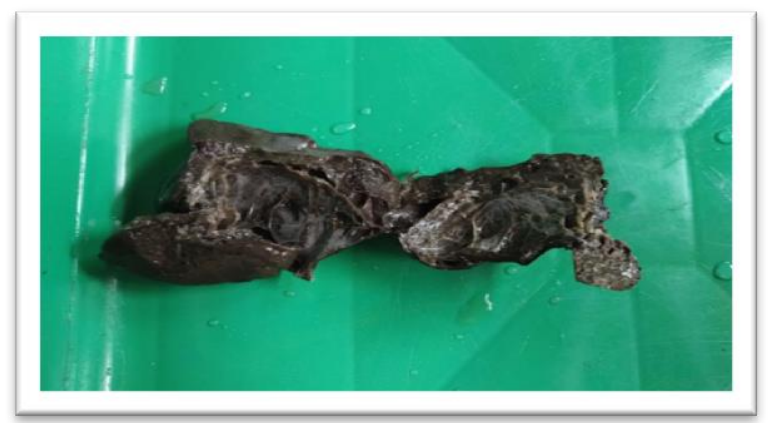

Figure 1: Cut section of lung showing unilocular cyst

The microscopy sections from the cyst wall showed a stratified cuboidal and ciliated columnar epithelium with focal intraluminal projections made of fibrous tissue. Cyst wall showed a few small and dilated cysts with similar lining forming a gland like pattern (anomalous bronchioles). Adjacent area showed intraalveolar hemorrhage and congestion. Sections from bullae showed fibrous tissue with hemorrhage. Cartilage was seen only in foci. Correlating with clinical history and histopathological findings, the diagnosis of Congenital Pulmonary Airway Malformation-Congenital Cystic Adenomatoid Malforation Type I was made. Following lobectomy, the child showed clinical improvement and was discharged home. Later she developed another episode of pneumonia that required hospital admission for 3 weeks. Currently she is thriving well.

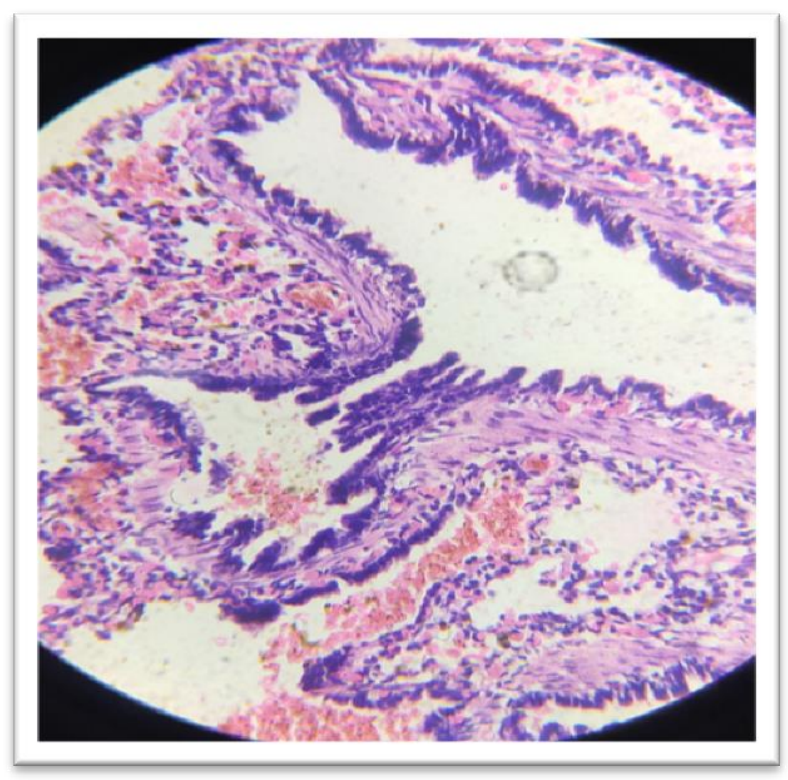

Figure 2: Microscopy showing cyst wall lined by stratified cuboidal and ciliated columnar epithelium with focal intraluminal projections made of fibrous tissue and cyst wall showing small and dilated cysts with similar lining

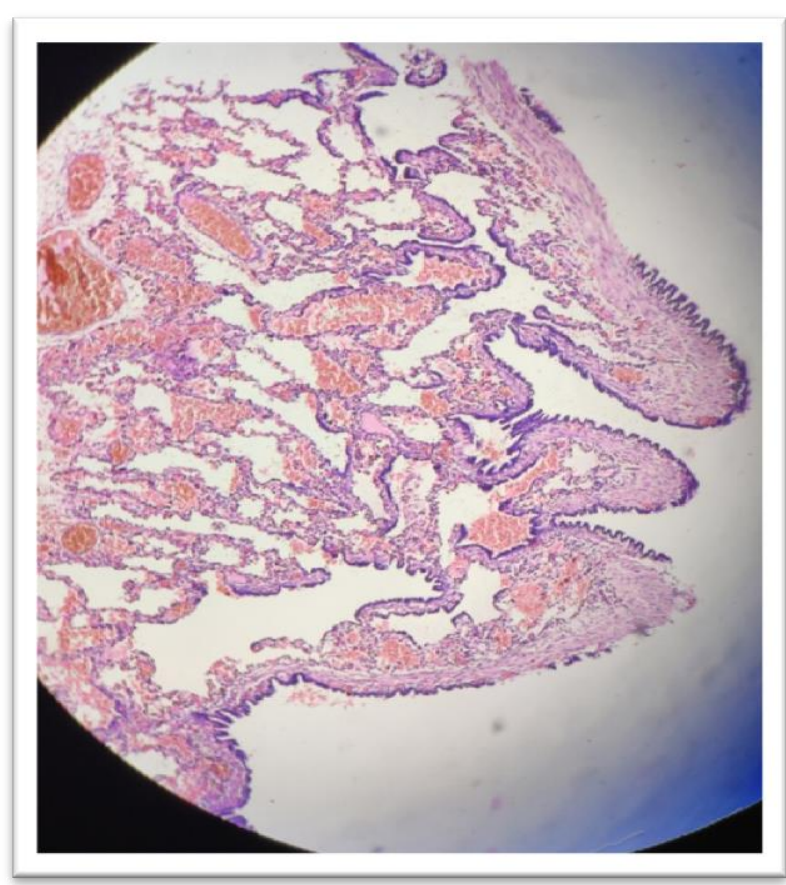

Figure 3: Microscopy showing cyst wall lined by stratified cuboidal and ciliated columnar epithelium with focal intraluminal projections made of fibrous tissue and cyst wall showing small and dilated cysts with similar lining

\section{DISCUSSION}

Congenital Cystic Adenomatoid Malformation of Lung (CCAM) is a rare non - hereditary developmental abnormality of lung with unknown etiology. This lesion was first described by Chi'n Tang in 1949 with males and females being equally affected. It is caused by anomalous fetal development of terminal respiratory structures, resulting in adenomatoid proliferation of bronchiolar elements and cyst formation leading to enlargement of the affected lobe. The clinical spectrum varies depending on the extent of malformation in the lung and associated conditions. The involvement of an entire lung is distinctly rare. A single lobe involvement is most common.

Most CCAMs occur in two distinct clinical settings: in a stillborn or extremely ill premature infant with anasarca or in a newborn infant who rapidly develops respiratory distress that is related to an expanding mass in one hemithorax. Less often the lesion is seen in older children and rarely in adults. The radiographic appearance of CCAM is variable. There may be a homogenous mass, multiple air or fluid filled cysts or a single or dominant radiolucent area. Spontaneous pneumothorax is an unusual presenting feature. The respiratory distress produced by the adenomatoid malformation is usually related either to tissue bulk or to air trapping within the lesion. In both instances, there is compression of the underlying normal lung parenchyma and even mediastinal shift. Circulatory symptoms occur because the mass obstructs the venacava and interferes with venous return to the 
Seena Susan Itty \& Sankar S; Saudi J Pathol Microbiol, Jan, 2022; 7(1): 29-32

heart producing anasarca. The lesion may be diagnosed by antenatal ultrasound examination, elevation of maternal serum alpha fetoprotein (AFP) is sometimes an associated feature.
Most CCAMs communicate with the normal tracheobronchial tree and air trapping presumably results from the usual lack of cartilaginous bronchi within the lesion. CCAM is classified into five types based on gross and histological features by Stock et al.,

\begin{tabular}{|l|l|l|}
\hline Type & Gross appearance & Microscopic appearance \\
\hline 0 & Solid & Bronchiole-like features, abundant cartilage \\
\hline 1 & Large, mutilocular cysts $3-10 \mathrm{~cm}$ & Broad fibrous septa, mucigenic cells, focal cartilage in 5 $-10 \%$ \\
\hline 2 & Small, uniform cysts $<2 \mathrm{~cm}$ & $\begin{array}{l}\text { Irregular proliferation of bronchiolar like structures, may contain striated } \\
\text { muscle }\end{array}$ \\
\hline 3 & Solid, bulky lesion;cysts $<0.2 \mathrm{~cm}$ & Irregular curved channels and small spaces lined by cuboidal epithelium \\
\hline 4 & $\begin{array}{l}\text { Large cysts, upto } 7 \mathrm{~cm}, \text { in } \\
\text { peripheral lung }\end{array}$ & $\begin{array}{l}\text { Thin walled cysts lined by type 1 pneumocytes and low columnar } \\
\text { epithelium, hypercellular stroma }\end{array}$ \\
\hline
\end{tabular}

Type 1 lesions are the most common accounting for over half the cases. Grossly they are composed of one or more cysts ranging from 3 to $10 \mathrm{~cm}$ in diameter. Microscopically, there are fibrous septa lined by pseudo stratified, ciliated columnar or cuboidal cells. Cartilagenous plates may be present focally in 5 to $10 \%$ of cases. In the more solid areas between the large cysts there are scattered bronchiole like structures that differ from true bronchioles in that they are irregularly sized and shaped and they are not accompanied by an aryery as would be expected in normal bronchioles. Plump mucus filled cells may occur in clusters and are found in upto one half of cases. Histochemically these cells resemble the goblet cells found in bronchial or intestinal mucous glands. Morphologic features of adenocarcinoma, usually the bronchoalveolar variant have been reported in association with Type 1 CCAM, but metastatic spread is extremely rare.

Type 2 lesions are the second most common variant and are characterized grossly by multiple evenly spaced cysts that are usually less than $2 \mathrm{~cm}$ in diameter. They may be associated with other congenital anomalies including renal agenesis, diaphragmatic hernia, cardiovascular anomalies and extralobar sequestration. Histologically they are characterized by uniformly distributedsmall cysts lined by cuboidal to ciliated columnar epithelium that superficially resemble ecstatic bronchioles, except that they are too numerous and, as in type 1 lesions are distributed randomly without an accompanying pulmonary artery branch. Striated muscle may be present in the septa or adjacent to the cysts.

Type 3 CCAMs are uncommon. They occur almost exclusively in males and are usually associated with maternal poly hydramnios. Babie with this lesion are often still- born or develop severe respiratory distress. They are characterized grossly by a firm or spongy mass of tissue in which macroscopic cyst are not readily found. Microscopically curved channels or microscopic cyst lined by cuboidal to low columnar epithelium are seen. Bronchial structures are not present.

Type 0 and Type 4 CCAMs were more recenty added to original three types of CCAMs and are extremely rare .Type 0 is felt to be a malformation of the proximal tracheo-bronchial tree and was previously termed acinar dysplasia or agenesis. It is incompatible life and is often associated with cardiovascular, renal and other abnormalities .Type 4 CCAM is considered to represent a malformation of the distal acinus. Tension pneumothorax occurs in some infants and is unique to this form of CCAM.

The pathogenesis of CCAM is unknown, but is thought to be related to defective lung development .The absence of cartilage in most cases suggest that there is an embryologic insult before the sixteenth week of intra uterine life by which time the cartilaginous bronchi are formed .Other histologic aspects of the lesion suggest that there may be a focal overgrowth of bronchioles with the suppression of alveolar development. There is also some evidence that te changes may occur secondary to bronchial atresia and other congenital and accured lung lesions. The variability in gross and microscopic appearance and a peripheral rim of more normal lung in most cases suggest that the lesion has some ability to undergo maturation.

The commonest presentation of CCAM in postnatal life is progressive respiratory distress including tachypnea, grunting, retraction, and cyanosis and in adults it usually presents as repeated chest infections, chest $\mathrm{X}$ ray, CT and MRI are helpful in diagnosis. The main complication in neonatal period is compression of the mediastinal structure producing cardiovascular compromise. In adult patient, CCAM is a nidus for pneumonia, abscess formation, fungal infection, spontaneous pneumothroax hemoptysis, air embolism, intra lobar sequestration and development of bronchogenic carcinoma. Serial antenatal sonographic evaluation, good obstetric care and delivery at a tertiary care centre are preferred plan of treatment for 
antenataly detected cases. Postnatal and in adult patients, lobectomy is the treatment of choice for symptomatic cases.

Prognosis also depends on stocker type, Type 1 lesions carry overall good prognosis. In Type 2 lesions it is the associated anomalies that determine the prognosis. Type 3 lesions carry bad prognosis as they are usually large and presence with cardiovascular compromise. Overall bilateral involvement, associated with hydrops and associated congenital anomalies carrypoor prognosis.

\section{CONCLUSION}

CCAM is a rare developmental malformation of lung. The routine use of prenatal ultrasonography has led to prenatal diagnosis and has provided great insight into the natural history of CCAM, which emphasizes the need of regular antenatal checkups for early detection and follow up.

\section{REFERENCES}

- Katzenstein and Askin's Surgical Pathology of Non-Neoplastic Lung Disease-major problems in Pathology, Fourth edition.

- Sfakianaki, A. K., \& Copel, J. A. (2012). Congenital cystic lesions of the lung: congenital cystic adenomatoid malformation and bronchopulmonary sequestration. Reviews in obstetrics and gynecology, 5(2), 85-93.

- Chikkannaiah, P., Kangle, R., \& Hawal, M. (2013). Congenital cystic adenomatoid malformation of lung: Report of two cases with review of literature. Lung India: Official Organ of Indian Chest Society, 30(3), 215-218.

- $\quad$ Patil, A. G., Karangadan, S., \& Kishore, V. (2015). Congenital cystic adenomatoid malformation of lung in fetus: report of two cases with brief review of literature. The Journal of Association of Chest Physicians, 3(2), 53.

- $\quad$ Sirageldin, M. K., \& Abdel, R. (2013). Congenital cystic adenomatoid malformation: A case report. Sudanese Journal of Pediatrics, 13(2). 